山्山FFRANÇAISE

$\supset \mathrm{DE}$

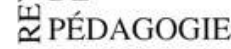

\section{Revue française de pédagogie}

Recherches en éducation

154 | janvier-mars 2006

La construction des politiques d'éducation : de nouveaux rapports entre science et politique

\title{
Le débat américain sur la certification des enseignants et le piège d'une politique éducative evidence-based
}

The American debate about teaching certification and the danger of evidencebased education policy

Die amerikanische Debatte über die Verbeamtung der Lehrer und die Falle einer evidence-based Erziehungspolitik

El debate americano sobre la certificación de los docentes y la trampa de una política educativa evidence-based

\section{Claude Lessard}

\section{OpenEdition \\ Journals}

Édition électronique

URL : http://journals.openedition.org/rfp/108

DOI : $10.4000 /$ rfp. 108

ISSN : 2105-2913

Éditeur

ENS Éditions

Édition imprimée

Date de publication : 1 mars 2006

Pagination : 19-32

ISBN : 2-7342-1039

ISSN : 0556-7807

\section{Référence électronique}

Claude Lessard, «Le débat américain sur la certification des enseignants et le piège d'une politique éducative evidence-based ", Revue française de pédagogie [En ligne], 154 I janvier-mars 2006, mis en ligne le 01 mars 2010, consulté le 20 avril 2019. URL : http://journals.openedition.org/rfp/108 ; DOI : $10.4000 /$ rfp. 108 


\title{
Le déb at a méric ain sur la certific ation deS en Seignants et le piège d'Une politique éd Uc ative evidence-based
}

\author{
$\mathrm{Clau}_{*}$. L ssar
}

Dans ce texte, nous analysons le vif déb a améric an à propos de la certific aion des enseignants du prim aire et du seCondaire. Il oppose des "professionnalistes" et des "dérégulationnistes". Ce débat est scientifique, car il pose la question de l'effet de l'enseignant sur l'apprentissage des élèves. Il est aussi idéologique en Ce que, tout en partageant le même souci du bien public en éducation, s'opposent ici deux modèles de qualité de l'enseignement: d'une part, un modèle professionnel axé sur une formation universitaire des enseignants de longue durée, Combinant une formation disciplinaire et une formation pédagogique, organisée et véCue en alternance, et d'autre part, un modèle déCentralisé de quasi-marché éducatif, de reddition de comptes et d'imputabilité des enseignants et des établissements. Enfin, le débat est politique, les partisans du seCond modèle Cherchant à briser le "monopole" des facultés d'éducation en matière de formation des enseignants et d'accès à l'enseignement. La référence à l'evidence-based policy et les rapports qu'elle révèle entre la science, la politique et l'idéologie sont abordés en Conclusion.

Descripte urs (TEE) : développement de l'éducation, États-Unis d'Amérique, initiation à ıa profession, formation des enseignants, professionnalisation.

\section{INTRODUCTION}

Les modes de légitim ation des sociétés modernes avanCées s'appuient surla "sCienCe " Conçue Comme un dispositif de production de savoirs sous forme de résultats de recherche empiriquement "solides" et par là "incontestables", ou encore sur des analyses de Contextes et de situations qui se présentent Comme "objectives" et "rationnelles", en même temps qu'elles semblent soutenues par le "sens Commun". Le texte qui suit entend illustrer Cette dernière affirmation, à partir d'un c a précis, so it le déb a actuel aux États-Unis à propos de la Certification des enseignants des écoles publiques (1). Ce débat voit s'affronter deux groupes qui se réclament de la science pour mieux mener leur combat idéologique et politique. Plus préCisément, les deux groupes reconnaissent la légitimité d'une politique éducative evidence-b aed, C'est-à-dire une politique qui " aide les gens à prendre des déCisions informées à propos de politiques, de programmes et de projets en mettant au cœur du développement et 
de l'implantation des politiques, la meilleure reCherche (evidenCe) disponible" (Davies, 1999). Une démarche de type evidence-b aed policy implique que soient mis en place des dispositifs de CompiIation de la recherche existante, un concept et des indicateurs de qualité de la recherche, et une Compréhension ou une interprétation de Ce que la recherche "dit" ou ne "dit pas". Dans le cas des sCienCes humaines et sociales, Cela est loin d'être évident, non seulement à cause des médiations idéologiques, mais aussi à cause de la difficulté des Consensus sur des indicateurs de qualité de la recherche et du caractère inCertain et in Complet du savoir des sciences sociales.

Dans ce texte, nous voulons donc analyser le vif débat, présentement en Cours aux États-Unis, à propos de la certification des enseignants du prim aire et $d u$ secondaire. Il oppose Ceux que, à la suite de Cochran-Smith et Fries (2 0 0), 3nous appellerons les "professionnalistes" et les "dérégulationnistes", et Ce débat est soutenu non seulement par des individus (universitaires, experts, cadres scolaires ou hommes politiques) m ais aussi par des fondations et des instituts privés pour qui Cette "Cause" est exemplaire d'un ensemble de questions plus larges, au sein du Champ éducatif, voire de l'ensemble de la société am éricaine.

Ce débat est scientifique, dans la mesure où il pose la question de l'effet de l'enseignant sur l'apprentissage des élèves. Plus préCisément, il pose la question de l'effet de la formation pédagogique et disciplinaire des enseignants sur la réussite des élèves: suivant la formule américaine $m$ al traduite en français, mais néanmoins courante, "l'enseignant fait-il une différence"? Si oui, quels facteurs produisent Cette différence ? La formation y est-elle pour quelque chose? Quelle form ation produit quel effet? Mais le débat déborde la science, notam ment en cherchant à imposer une manière quelque peu réductrice de poser Cette question et une seule et unique méthodologie pour y épondre.

Le débat est aussi idéologique en ce que, tout en partageant le même souci du bien public en éducation, s'opposent ici deux modèles de qualité de l'enseignement : d'une part, un modèle professionnel axé sur une formation universitaire des enseignants de longue durée, Combinant une formation disciplinaire et une formation pédagogique, organisée et véCue en alternance, et d'autre part, un modèle déCentralisé de quasi-marché éducatif, de reddition de Comptes et d'imputabilité des enseignants et des établissements. On peut aussi affirmer sans trop se tromperque le débat oppose deux Conceptions d'un enseignant de qualité : d'un Côté, un enseignant dit "Compétent", et de l'autre, un enseignant dit "efficace". Les tenants de l'enseignant efficace jugent de la plus grande importance to ut Ce qui contribue à une valorisation inconditionnelle des résultats mesurés. Par opposition, les tenants de l'enseignant Compétent résistent $\operatorname{tant}$ bien que $m$ al à une responsabilisation qui leur semble excessive des enseignants et à une ConCeption qui leur apparaît réduCtrice de la $\mathrm{m}$ ission de l'enseignement. Mais tout porte à croire que ces derniers sont surla défensive, essayant de limiter les avancées des premiers, bien branchées surl'air du temps politico-idéologique et surde puissants réseaux.

Enfin, le débat est politique en ce que les partisans $\mathrm{du}$ second modèle CherChent à briser le "monopole" des facultés d'éducation en matière de formation des enseignants et d'accès à l'enseignement. Ils proposent également des programmes alternatifs de formation, des voies multiples de Certification et d'accès au métier, et ils voudraient déplacer une partie du pouvoir de l'université vers les établissements et les directions d'écoles.

Nous entendons dans ce texte analyser ce débat, dans un premier temps en présentant les protagonistes, les agendas qu'ils épousent et le Contexte de Concurrence entre les groupes en présence pour le Contrôle de la politique éducative américaine. Dans un second temps, nous nous attacherons au débat scientifique proprement dit, en identifiant les données disponibles et débattues, les zones de consensus et de discussion ainsi que les stratégies d'argumentation utilisées pour tenter de vaincre l' "adversaire " et de "remporter la victoire». Enfin, en Conclusion, nous réfléchirons surles caractéristiques du débat, notamment surla référence à l'evidence-b aed policy et surles apports qu'elle révèle entre la science, la politique et l'idéologie.

\section{LES PROTAGONISTES : DES FONDATIONS PHILANTHROPIQUES INFLUENTES, DES UNIV ERSITAIRES ET DES CHERCHEURS ENGAGÉS}

Les fondations et les instituts privés Constituent des interfaces entre le politique, le monde du savoir et les divers univers d'action sociale. Historiquement, ils ont soutenu les arts, des bibliothèques et des recherches; ils ont épousé des causes, Comme 
I'enseignement public de qualité, et par leurs modes de travail et la qualité de leur réflexion, ont pu Contribuer à influer surl'état sinon de l'opinion publique en général, $d u$ moins de Celle qui Compte en m atière d'influence et de prise de déCision. Au Cours des dernières déCennies, les fondations sont passées à l'action et se sont engagées surdes terrains préCis. Par exemple, C. E. Finn et D. RavitCh, de la Fordham Foundation, ont mis surpied en 1981 un réseau d'exCellence - Educ aonal Excellence Network (EEN) -, une organisation sponsorisée par la fondation et axée sur des réformes éducatives "fondées" (sound) et la diffusion d'informations et d'idées "valides" à propos de l'éducation. Ce réseau se caractérise par une volonté de hausser les exigences (standards) de formation et d'assurer un Contenu académique fort, ainsi que par l'acceptation d'une imputabilité "pure et dure"(tough-minded ac ountability).

Le débat qui nous intéresse oppose deux groupes d'intellectuels universitaires (Certains acteurs importants des sciences de l'éducation américaines, par exemple Linda Darling-Ham mond (2), d'autres davantage liés à la culture disciplinaire universitaire, $p$ ar exemple Diane Ravitch (3)), des hommes et des femmes politiques (des gouverneurs, des membres des deux grands partis nationaux, menant carrière dans les institutions politiques fédérales ou dans les instances politiques des divers États), des cadres scolaires (surintendants et cadres supérieurs), des hommes d'affaires, et des chercheurs et des analystes à l'emploi des fondations engagées dans le com bat.

De plus, si les fondations sont des entités distinctes largement à cause de leur histoire et de la $m$ arque du fondateur donateur, elles sont en lien les unes avec les autres, du moins parmi Celles qui partagent des "sensibilités politiques Communes". Cela est particulièrement observable dans Ia Composition des Conseils d'administration et parmi le groupe des "senior associates". C'est ainsi que Chester E. Finn (4) est rédacteur de la revue EduC aion Next, publié par la Hoover Institution, tout en étant le président et le direCteur général de la Fordham Foundation. D. Ravitch est à la fois membre de la Brookings Institution et de la Fordham Foundation. La revue Educ aion Next, porte-parole de l'agenda de la dérégulation, est soutenue, entre autres, par le Manhattan Institute (New York), la Fordham Foundation et la Hoover Institution. Il y a donc un réseau de personnes et d'organismes qui se reconnaissent Comme partageant le même agenda ou ayant une même vision politique, voire des intérêts Communs.
L'étude des fondations et des instituts privés, en tant qu'interfaces, soulève plusieurs questions. Parmi Celles-Ci, quatre sont particulièrement importantes: 1) Quels agendas de réformes épousent-ils? 2) Quels savoirs et quels Champs de Connaissance sont pertinents et méritent d'être soutenus et développés dans le $c$ are du travail des fondations et des instituts dans le dom aine des politiques publiques? 3) Qui sont Ces experts produCteurs d'analyses et éventuellement de politiques structurant le déb a public en éduc aion? et 4) Quelle est la place de l'expertise dans le déb apublic et dans le processus de prise de déCision démocratique ? Dans Ce texte, nous aborderons les deux premières questions, à partir du cas à l'étude, so it Celui de la politique am éricaine à propos de la Certification des enseignants du primaire et du secondaire.

\section{LES DEUX AGENDAS DE RÉFORME : LA DÉRÉGULATION ET LA PROFESSIONNALISATION DE LA FORMATION DES ENSEIGNANTS}

Pour les fins de notre analyse, il importe de nous attarder quelque peu surle manifeste de la Fordham Foundation intitulé The Teachers We Need and How To Get More of Them : A Manifesto (1999). Ce manifeste a été signé par une Cinquantaine de personnalités, dont C. E. Finn, D. RavitCh et plusieurs autres associés à diverses fondations et instituts Conservateurs, Comme la Heritage Foundation, le Hudson Institute, le Goldwater Institute, le Pionner Institute, et le Manhattan Institute. Le $m$ anifeste reprend l'élément de base du diagnostic scolaire fait en 1983 par le rapport $A$ Nation at Risk: les écoles américaines sont sous-performantes et le problème ne sera pas résolu tant qu'il n'y aura pas dans Chaque classe d'excellents enseignants. Selon le manifeste, il est clairement démontré que la variable la plus importante dans l'apprentissage des élèves, C'est la qualité de l'enseignant. Et toutes les réformes éducatives échouent parce qu'elles sont incapables de garantir que de plus en plus d'enseignants possèdent le savoir et les capacités nécessaires pour aider tous leurs élèves à apprendre. Nous savons que les enseignants "font une différence". Ce qu'il importe de savoir, soutient le manifeste, C'est Comment les former, les attirer, les évaluer et les retenir dans l'enseignement. Or, Ia formation des enseignants américains souffre de sérieuses carences, elle n'a pas bonne réputation, y Compris auprès des enseignants eux-mêmes; de plus, les enseignants sont 
insuffisamment formés dans les matières qu'ils ont à enseigner. Aussi, on est in C aable d'attirer des étudiants en formation des maîtres possédant d'excellents dossiers scolaires (the best and the brightest) et on semble inc aable de retenir un nombre suffisamment élevé de Celles et Ceux qui sont parmi les meilleurs étudiants. Le système actuel de formation, Conclut le manifeste, est en somme inc aable de répondre au double défi de la qualité et de la quantité.

La réponse réCente d'un Certain nombre d'États à Ce double problème a été de resserrer les exigences de Certification : plus de Cours, des tests standardisés et des stages prolongés. Cette réponse, selon le manifeste de la Fordham Foundation, ne peut être efficace, car, du pareil au même (more of the same), elle renforce le statu quo (C'est-à-dire le pouvoir des facultés d'éducation et des bureaucrates), alourdit, standardise et uniformise davantage le système de Certification, impose une orthodoxie professionnelle plus proche des idéologies et des modes que des résultats de recherche solides, et est Coûteuse puisqu'elle mène inévitablement à un accroissement de la scolarité avant l'insertion dans le métier. De plus, Cette réponse n'est pas justifiée, car la soi-disant base de Connaissances qui fonde la prétention des sCiences de l'éducation à assumer la responsabilité pédagogique et pratique de la formation des enseignants, n'est pas très solide (elle est "shaky»). Et lorsqu'elle existe dans un secteur donné, les éColes de formation de maîtres ont tendance à ne $p$ as en tirer les nécessaires Conséquences, puisque cela leur apparaît Contraire à leur idéologie.

Il faut donc penser à autre chose, selon la Fordham Foundation: "the time has come to Consider radi$C$ ay different policies to boost the quality of teaching in U.S. schools" (1999, p. 8). Cette autre approche n'est ni fondée sur la science ou sur l'idéologie; elle relève plutôt du sens Commun. En effet, Celui-Ci nous dit que s'il faut déréguler la profession enseignante, en ouvrir l'accès à une plus grande diversité de candidats, et permettre aux chefs d'établissement d'embaucher et d'évaluer qui leur semble compétent, alors il importe de s'assurer que les écoles et leurs administrateurs soient imputables de leurs déCisions et surtout de l'apprentissage et de la réussite des élèves dont ils sont responsables, $m$ ais également, mettre fin au monopole des sCiences de l'éducation. En somme, affirme le manifeste, plus de liberté pour davantage de résultats: "School level $m$ anagers are in the best position to know who teaches well and who teaches $b$ aly. They have ac oss to far more signific at information that state licensing boards and government agencies. They should be empowered (and, if need be, trained) to appraise each teacher's singular package of strenghts and weaknesses rather than having distant bureaucracies decide who should be on their team. Once hired, teachers should be evaluated on the only $m$ easure that really $m$ atters : whether their pupils are learning " (1999, p. 9).

Ce qu'il faut faire donc, suivant Ce «sens Commun", est apparemment simple et direct. Cela tient en quatre points :

- les États devraient développer des systèmes d'imputabilité des écoles axés surles résultats ; Ces systèmes devraient porter tant surles écoles, les enseignants que les élèves;

- les États devraient autoriser les directions d'éColes à prendre des déCisions en matière de personnel enseignant et de gestion des ressources humaines de l'établissement dont ils assument la responsabilité ;

- les États devraient m aintenir un minimum de régulation du personnel enseignant afin de s'assurer que dès l'entrée, les enseignants ne puissent causer de préjudice sérieux aux élèves : Celle-Ci devrait Comprendre des vérifications de leur passé, l'exigence d'un baccalauréat (IiCence) disciplinaire, et des examens imposés par l'État $d$ ans les matières d'enseignement;

- les États devraient aussiouvrir de nouvelles avenues pour l'enseignement, encourager la diversité et la liberté de choix entre plusieurs dispositifs de préparation à l'enseignement, et accueillir dans l'enseignement un bassin plus large de personnes talentueuses et bien formées désireuses d'enseigner ( $m$ ême si Celles-Ci ne sont pas formées en sciences de l'éducation).

Il est clair que l'administration américaine actuelle soutient la position des dérégulationistes. On n'a qu'à lire le rapport du secrétaire d'État à l'Éducation intitulé Meeting the Highly Qualified Teachers Challenge $(200)$ \$our dissiper tout doute à Ce propos. En effet, dans la politique présidentielle No Child Left Behind (qui a maintenant force de loi), G. W. Bush s'est engagé à Ce que Chaque classe américaine soit enseignée par un enseignant «qualifié ». La définition légale du gouvernement fédéral de ce qu'est un enseignant qualifié met l'accent prioritaire sur la Connaissance disciplinaire (5). Le secrétaire d'État à l'Éducation, R. Paige, tire les conséquences de Ce retour en force des disciplines: la formation 
pédagogique, dans les programmes universitaires en éduc aon, devrait être optionnelle et les programmes alternatifs, nés dans l'urgence et pour Contrer la pénurie, devraient être dorénavant des solutions permanentes, adaptées à un marché de la formation désormais dérégulé, sans monopole universitaire, et surtout, financés par l'État.

L'agenda est aussi en cours de réalisation, pour autant qu'il participe du nouveau paradigme des politiques publiques en éducation, so it Celui qui met l'accent surla responsabilisation des établissements et des acteurs locaux dans l'apprentissage des élèves, l'obligation de résultats et l'imputabilité des acteurs locaux.

Pour sa part, l'agenda de la professionnalisation de l'enseignement est soutenu par la Carnegie Corporation et la Rockefeller Foundation de New York, les Pew Charitables Trusts, la Ford Foundation, et le De Witt Wallace Reader's Digest Fund (CochranSmith \& Fries, 2001, p. 3). Cet agenda a été d'abord problématisé, formulé et justifié en 1986 dans le Rapport A Nation Prepared: Teachers for the Twenty-First Century, fin an Cé p ar la Carnegie Corporation de New York, à travers son programme intitulé le Carnegie Forum sur l'éduc aion et l'éConomie. L'agenda de professionnalisation qu'il propose est en cours de réalisation, $d u$ moins en partie et dans Certains États et districts scolaires, grâce aux efforts Conjoints du National Council for the Accreditation of Teacher Education (NCATE), revivifié à partir de 1991, le National Board for Professionnal Teaching Standards (NBPTS), Créé en 1987, et l'Interstate New Teacher Assessment and Support Consortium (INTASC), lui aussi mis surpied en 1987.

Il importe aussi de mentionner la National Comm ission on Teaching and America's Future sur la form ation des enseignants dont le rapport a été rendu public en 1996 et qui a servi de base à la Construction de partenariats entre une quinzaine d'États et la Commission nationale sur l'enseignement et l'avenir des États-Unis. Linda Darling-Hammond en a été la secrétaire et la direCtrice générale. L'agenda est aussi en voie de réalisation dans les universités qui, dans la foulée des rapports du Holmes Group (Lessard, 200 )3 CherChent à améliorer substantiellement leurs programmes de formation des maîtres, en rehaussant les exigences et les seuils de réussite, en allongeant la formation (quatre ou cinq ans) et dans un nombre croissant de cas, en créant des maîtrises en enseignement, et aussi en mettant sur pied des écoles de formation Clinique - les Professionnal Development Schools (PDS).
Ainsi s'affrontent deux agendas de réforme de la formation des maîtres. Iss renvoient à des visions différentes de l'enseignant, de sa mission, et de la réforme de l'éCole.

\section{LE CONTEX TE DE CONCURRENCE IDÉOLOGIQUE ET LA GENÈSE DU DÉBAT ACTUEL : A NATION AT RISK (1983) ET A NATION PREPARED (1986)}

On peut faire remonter la genèse du débat actuel au début des années 1980 et au Célèbre rapport de la National Commission on ExCellence in Education, intitulé $A$ Nation at Risk. Considérons Ce rapport Comme représentant la droite américaine et incarnant la fin d'une époque - Celle des Trente glorieuses et de la démocratisation de l'école et de la Culture et le début d'une autre, marqué par le retour en force d'un discours résolument éConomiste, axé sur l'efficacité et la productivité de l'industrie de l'enseignement, rendue nécessaire pour une Concurrence plus vive entre les nations du monde dans le Contexte de la globalisation économ ique.

La Commission ne Comprenait auCun représentant d'associations professionnelles ou syndicales d'enseignants, ni de porte-parole des facultés d'éducation. Pouvant s'appuyer sur une perCeption assez largement répandue au sein de l'opinion publique américaine, du moins de l'avis du secrétaire d'État à l'Éducation d'alors, à l'effet que l'école publique américaine n'était pas à ıa hauteur des attentes de la population (6), Ia Comm ission avait pour mandat d'apprécier la qualité de l'enseignement et de l'apprentissage dans les écoles, les collèges et les universités et de Comparer les éColes et les Collèges am éricains avec Ceux des autres nations développées. C'est Cependant surtout de l'école secondaire - le high school-que la Commission a traité.

A Nation at Risk prend ses distances par rapport aux discours qui ont dom iné la scène éducative américaine tout au long $d u X X^{e}$ siècle : pour la Comm is sion, l'enjeu majeur n'est pas la contribution de l'école publique à l'intégration des immigrants et à la Construction de la nation américaine, sa mission de développement d'une Citoyenneté démocratique, l'écart entre une valeur méritocratique et son fonctionnement réel, notamment dans son traitement des minorités "visibles", ou encore sa capacité d'innover selon les idéologies pédagogiques des années 1960 . Bref, l'enjeu majeur ne renvoie $p$ as aux fonctions 
sociales et politiques de l'école; il porte plutôt sur la performance proprement scolaire de l'école, Celle-Ci étant fonction des apprentissages réalisés par les élèves, mesurés $p$ ar des tests $s$ tandardisés et perÇus Comme direCtement responsables de la Compétitivité de l'éConomie améric ane sur la scène mondiale. Il y a iCi à l'œuvre une réelle réduCtion du Champ d'intérêt et de préoc opation vis-à-vis de l'école : Cest sa performance, définie en termes de résultats à des tests standardisés, qui est questionnée, les dimensions plus larges et plus traditionnelles de sa $\mathrm{m}$ ission étant reléguées au statut de discours anciens, Certes généreux m ais désormais secondaires, Cestà-dire en somme de moins en moins pertinents.

Le rapport $A$ Nation at Risk a été Critiqué pour son discours alarmiste sur l'état des écoles et les acquis des élèves. Aussi, la thèse du déclin renvoie à un prétendu âge d'or, Iargem ent im ag in aire. La Com m ission a aussi essuyé de nombreuses Critiques à propos de la recherche et des données quantitatives utilisées et notam ment de la grande Confiance que lui inspiraient les tests Comme le Ceux du College Board, Cux du National Assessment of Educational Progress et les tests internationaux. Comme le reConnaît aujourd'hui D. Ravitch (2 $00,3.35)$, les données quantitatives disponibles à propos de la qualité de l'éducation américaine sont limitées et partielles, et l'on sait peu de Choses surles inégalités de réussite.

Que dit la commission à propos des enseignants ? Le message à leur propos se présente de manière simple et directe : il faut en recruter de meilleurs et Combler les besoins, notamment $d$ ans les matières à fortes retombées technologiques et éConomiques - les sciences, les m athém atiques. À Cette fin, Ia formation des maîtres doit s'assurer que les futurs enseignants soient capables de rencontrer des exigences académiques élevées, qu'ils démontrent une aptitude à enseigner et qu'ils m aîtrisent la discipline qu'ils désirent enseigner. Et les facultés d'éducation devraient être jugées en fonction de la qualité de leurs diplômés. Aussi, puisque la m aîtrise des savoirs disciplinaires est en définitive plus importante que la pédagogie, afin de Combler la pénurie d'enseignants, recrutons pour l'enseignement plus largement que parmi les seuls diplômés des facultés d'éducation. Rendons possible l'accès à l'enseignement, après une formation pédagogique minimale (acquise possiblement dans l'école même du premier emploi, sous la guidance d'enseignants Chevronnés), à des diplômés universitaires de sCiences ou de mathématiques, à des ingénieurs ou à des scientifiques en exercice ou retraités, mais intéressés par l'enseignement.
D'ailleurs, Ia Comm ission est d'avis que des Centres scientifiques importants ont la capacité de former ou de recycler des scientifiques pour l'enseignement. Au nom de la pénurie réelle ou Construite, la porte est ainsi ouverte à une révision de la politique de for$m$ ation des maîtres et au mandat Confié à Cet effet aux facultés d'éducation.

Évaluant le Chemin parcouru depuis vingt ans, Finn estime que, suite au rapport de la Commission sur l'excellence en éducation, des transformations significatives de l'école américaine ont été réalisées, notamment aux plans du Curriculum et des exigences (standards) pour les élèves. En Ce qui ConCerne les enseignants, selon lui, peu de Changements ont vule jour. Pourquoi? Deux raisons principales sont évoquées: la forte résistance de l'establishment éducatif (les syndicats d'enseignants, les institutions de formation de maitres et les bureaucraties éducatives des États) et l'émergence, soutenue par Ces mêmes groupes résistants, d'un discours de nature professionnalisante. Pour Finn, Ce discours, matérialisé dans le rapport $A$ Nation Prepared, produit en 1986 par le Task Force on Teaching as a Profession de la Carnegie Foundation, est Contraire à Celui de A Nation at Risk: "A Nation Prepared's most notable feature was a subtle yet profound change of focus: from teachers as instruments of school improvement to teachers as shapers of school improvement. From teachers as means to teachers as ends. From teachers as staff in an education system run by others, to teachers as key decision makers about the purpose and operations of the system itself. One might almost say from teachers as workers to teachers as bosses" (2003, p. 218).

La lecture que Finn propose du rapport $A$ Nation Prepared est juste. En effet, si Ce rapport du groupe de travail sur l'éducation et l'économie de la Carnegie Foundation part des mêmes Constats que Ceux du rapport $A$ Nation at Risk, encore qu'il les interprète différemment, il n'en tire pas to ut à fait les mêmes Conclusions. Pour Ces auteurs, si le système scolaire am éricain est déficient, Ce n'est pas tant qu'il soit en déclin ou médiocre; C'est plutôt qu'il est inadapté au Contexte actuel et surtout incapable de relever les défis de la soCiété et de l'éConomie du savoir. Il ne s'agit pas de restaurer un prétendu âge d'or d'antan, il importe plutôt de résolument regarder devant soi et agir en fonction des intérêts bien Compris de la nation. Si les États-Unis veulent demeurer une puissance économique de premier plan, s'ils désirent Conserver leur niveau et leur qualité de vie supérieure, dans un Contexte de mondialisation économique où 
les pays en développement réCupèrent de plus en plus d'emplois peu spécialisés, il importe de former une main d'œuvre de plus en plus qualifiée, ain de maintenir un avantage Compétitif au plan des seCteurs d'activité et des emplois de haute valeur. Ce discours économiste rend impérieux la réussite scolaire pour tous : "If our standard of living is to be $m$ aintained, if the grow th of a permanent underclass is to be averted, if democracy is to function effectively into the next Century, our schools must graduate the vast $m$ ajority of their students with achievement levels long thought possible for only the privileged few $"(1986, \mathrm{p} .3)$.

Certes, pour y arriver, il faut revoir à la hausse les exigences du Curriculum secondaire américain, $m$ ais Celui-Ci ne peut générer les fruits escomptés que si les enseignants sont des professionnels de qualité : "the key to suc oss lies in creating a profession equal to the task - aprofession of well-educ aed teachers prepared to assume new powers and responsabilities to redesign schools for the future." (1986, p. 2). Les Changements souhaités par A Nation Pre$p$ ared Constituent des dimensions de la professionnalisation de l'enseignement et de la formation des enseignants. Il s'agit de Créer une sorte d'ordre professionnel national, Chargé de définir et de superviser le respect de "standards" nationaux de Compétence professionnelle (un National Board for Professional Teaching Standards), restructurer les écoles et revoir l'organisation du travail enseignant de sorte que les enseignants soient Collectivement autonomes au plan des moyens et des dispositifs pédagogiques privilégiés et responsables des résultats des élèves, introduire progressivement un plan de carrière hiérarchisé, et mettre sur pied une formation des maitres Comprenant un baccalauréat dis Ciplinaire et une maîtrise en enseignement, ainsi qu'améliorer les Conditions statutaires (salaires, ré Compenses, etc.). Notons que Ces mesures seront pour l'essentiel reprises de 1986 à 1996 par les divers rapports du Groupe Holmes et que Certaines seront partiellement $m$ ises en application (Lessard, 200 )3

Les deux rapports sont en quelque sorte Contem porains : si le premier date de 1983 , le second est de 1986. Émergent donc deux discours et deux agendas (7) différents et en bonne partie Contradictoires sur la place et le pouvoir des enseignants dans l'éCole et dans les déCisions ConCernant l'enseignement dispensé. Mais il y aurait plus en cause. En effet, deux visions de l'enseignement et de l'apprentissage s'affronteraient : une vision plus traditionnelle, axée sur la transmission des Connaissances et bien vue des parents, et une autre vision, plus moderne, so Cio-ConstruCtiviste, axée sur l'apprentis. sage et sur l'enseignant Comme facilitateur, entrấneur et mentor. Cette seconde vision serait épousée par l'establishment éducatif. La première appelle un redressement de la situation, la $m$ ise en place de mesures administratives et pédagogiques plus efficaces ; la seconde Commande une "restructuration", voire une "reculturation" de l'école. "The key difference is that the Carnegie team sought to shift more Control (and resources) into the hands of educators and their interest groups, while the Excellence Commission seemed Content with the "Civilian Control" arrangements that traditionally characterized the system's governance " (Finn, 200 ,3p. 221).

On pourrait à juste titre penser que Finn, dont les appartenances et les orientations idéologiques néolibérales sont Connues et clairement affichées, adicalise les différences entre les deux Courants et les deux groupes, parce qu'il cherche à montrer que les groupes qui soutiennent la professionnalisation de l'enseignement ont détourné le vent de réforme qui soufflait aux États-Unis au début des années 1980 en faveur d'une plus grande efficacité et performance des écoles, vers une plus grande reconnaissance statutaire des enseignants et un plus grand pouvoir de l'establishment éducatif sur l'école et sur l'organisation de la formation des maîtres. Finn n'a pas tort de Considérer l'idéologie de la professionnalisation Comme Concurrente de Celle sur l'efficaCité et surla performance. La première épouse une Conception plus large de la réussite de l'élève et de l'efficience de l'établissement et elle estime que la $m$ ission de l'enseignant dépasse sa capacité à faire augmenter les résultats d'un groupe d'élève sur des tests standardisés. Les professionnalistes estiment eux-mêmes que l'école de l'avenir, Celle qui pourra insérer ses diplômés dans la so Ciété du savoir, devra développer Chez Ceux-Ci des Compétences et des Connaissances de haut niveau, qui ne sont pas facilement mesurables, en tout cas pas par des tests standardisés Centrés surdes acquisitions et non sur des capacités. Les professionnalistes ont donc une Conception plus large et ouverte de l'apprentissage, alors que les dérégulationistes le ramènent à ce qui peut être quantitativement mesuré à l'échelle d'un systè $\mathrm{m}$ e.

Par ailleurs, pour les dérégulationistes, Ce qui est en cause c'est la Contribution des deux agendas de réforme à l'amélioration des apprentissages des élèves. D'où la question qu'ils posent : quelles données de recherche soutiennent l'un ou l'autre agenda? 
A t-on vraiment prouvé qu'un enseignant diplôm é d'une faculté d'éduc aon a un effet plus positif sur les apprentissages des élèves qu'un enseignant sans formation pédagogique ( $m$ ais avec néanmoins une formation disciplinaire universitaire)? Comment s'y prend-t-on pour démontrer Cela?

\section{LE DÉBAT SCIENTIFIQUE : QUELLE RECHERCHE DE QUALITÉ POUR QUELLE PROPOSITION DE POLITIQUE?}

Malgré des divergences d'orientations et de valeurs, l'accord entre les parties au débat existe sur quelques points majeurs qui structurent Considérablement la discussion scientifique. Le premier point d'accord porte sur l'importance de l'evidence-b aed policy, C'est-à-dire sur l'idée que la politique éducative doit s'appuyer le plus possible, voire découler directement, de la "recherche»de qualité, et non pas de l' "idéologie ". Le second porte sur le lien nécessaire entre les caractéristiques des enseignants (dont la formation) et l'apprentissage des élèves : un bon enseignant fait progresser ses élèves surle plan des Connaissances et des capacités, et Cela se mesure. Avec Ces deux éléments, ainsi qu'on tentera de le montrer dans les paragraphes qui suivent, le débat, dans ses aspects scientifiques, devient à la fois trè spéCialisé et très polarisé, masquant ainsi une importante zone d'accord.

On peut présenter sous la forme de quatre propositions les éléments essentiels du débat scientifique.

Première proposition: la recherche montre que les enseignants "font une différence". Une fois Contrôlés les effets du milieu et les caractéristiques des élèves, l'enseignant, plus que toute autre caractéristique de l'école, a un effet significatif sur l'apprentissage des élèves et Cet effet est durable. Les deux parties au débat reconnaissent le bien-fondé de Cette proposition et estiment que bon nombre de recherches empiriques de qualité en présentent une preuve Convaincante.

Deuxième proposition : la recherche fournit des éléments de réponse à la question suivante : quelles sont les caractéristiques de l'enseignant qui font une différence ? Ces éléments sont in Complets, car tout n'est pas mesurable (Laczko-Kerr \& Berliner, 200 )2 et l'enseignement de qualité demeure mystérieux (Goldhaber, 200 )2; néanmoins, ils sont utiles pour orienter les politiques. Les deux parties au débat reconnaissent l'importance démontrée d'une formation disciplinaire (mesurée par un diplôme de majeur ou de baccalauréat disciplinaire), de l'expérience (jusqu'à un Certain niveau, au-delà duquel elle ne joue plus ou peu) et de l'habileté verbale. En ce qui Concerne la formation disciplinaire, de nombreuses études surl'enseignement hors-Champ au seCondaire montrent hors de tout doute que l'apprentissage des élèves, notamment dans les $m$ atières scientifiques, est mieux assuré par des enseignants possédant une formation disciplinaire pertinente. Quant à l'effet de l'habileté verbale, il a été mesuré la première fois dans la Célèbre étude de Coleman (1966) au Cours des années 1960 et plusieurs États américains ont par la suite intégré dans leurs tests de Certification des enseignants une mesure de l'habileté verbale (tout comme d'ailleurs des examens de la compétence dis Ciplin aire).

Ce qui ne fait pas Consensus, C'est le statut accordé à Ces variables. Prenons l'exemple de la formation disciplinaire. Ainsi que l'affirme Darling-Hammond (2 0 0), 2il serait ridicule de prétendre que la Compétence disciplinaire est sans importance, mais selon elle, il est également ridicule de soutenir que la Connaissance de l'enseignement et de l'apprentis. sage, ainsi que la possibilité structurée d'apprendre à enseigner sous la supervision d'un enseignant Chevronné dans le cadre d'une formation pratique bien encadrée, sont sans conséquence. Pourtant, les dérégulationistes, estimant que la recherche "sérieuse " ne soutient véritablement que l'importance de la formation disciplinaire, sont d'avis que les politiques ne devraient rendre obligatoire que Cette formation, et pas l'autre de nature pédagogique, ainsi reléguée au statut d'optionnelle ou de facultative. Les dérégulationistes affichent iCi un point de vue assez simpliste de la relation entre la recherche et l'élaboration des politiques: Celles-Ci ne doivent pas aller au-delà de ce que la recherche démontre. Ce qui n'est pas prouvé empiriquement, ou de façon incertaine et ambiguë, ne doit pas être objet de législation. Il faut dire que les dérégulationistes cherchent à défaire un arrangement institutionnel, qu'ils présentent toujours Comme le monopole des facultés d'éducation sur la formation. Il est donc de bonne guerre de tenter de montrerque la recherche "sérieuse " ne justifie pas le maintien de Ce monopole.

Notons qu'il est empiriquement difficile de séparer les effets des deux types de formation, disciplinaire et pédagogique, car s'il est vrai qu'il se trouve dans les écoles américaines des enseignants avec une 
formation disciplinaire et sans form ation pédagogique, l'inverse est à peu près impossible. En effet, un diplômé d'une institution améric ane de formation de m aîtres a reçu une formation à ıa fois disciplinaire et pédagogique. De telle sorte que lorsqu'on mesure l'effet d'un diplôme d'éducation sur l'efficience des enseignants, Ce diplôme révèle l'influence potentielle des deux formations inextricablement liées. Pour les dérégulationistes, le fait qu'on n'ait $p$ as isolé l'effet de la formation pédagogique constitue une faiblesse importante de la preuve présentée par les professionnalistes. Ces derniers répliquent en mettant de l'avant une Conception plus large de l'expertise enseignante, disons une vision multidimensionnelle et moins réductrice de la qualité de l'enseignement. Certes, ils retiennent et intègrent les variables que les dérégulationistes estiment sCientifiquement in Contournables, $m$ ais ils les placent dans un ensemble plus Complexe et riche.

Il y a donc un débat surl'importance d'une formation pédagogique préalable à l'exercice du métier, et par implication, sur la nécessité de faire de Celle-Ci une exigence de Certification des enseignants. Comment la recherche peut-elle Contribuer à trancher Cette ques. tion? Dans le Contexte américain, une sous-question de recherche est lapidement associée à la première : s'il est nécessaire de former les futurs enseignants dans quelque chose d'autre que les Contenus d'enseignement, les programmes universitaires de baccalauréat en éducation sont-ils plus performants que des programmes "alternatifs", plus Courts, dispensés quelques semaines avant la prise de fonction et souvent gérés par les autorités scolaires locales?

Troisième proposition: pour que la recherche empirique soit en mesure de Contribuer à éclairer Ces questions, elle doit être de qualité. Qu'est-Ce qu'une recherche de qualité ? Pour le Compte de la Abell Foundation, K. Walsh (2 0 10) a produit une analyse secondaire de la recherche soutenant l'importance de la formation pédagogique et de la Certification des enseignants. Cette analyse a porté sur 175 études empiriques réalisées au Cours des Cinquante dernières années et mentionnées par les professionnalistes dans leur argumentaire en faveur de la formation pédagogique obligatoire. Parmi Ces études, Walsh a sévèrement Critiqué la recherche effectuée par Darling-Hammond (2 000$) .0$ Cette analyse de Walsh est souvent Citée et Darling-Ham mond a senti le besoin de répliquer dans un texte d'une Cinquantaine de pages (2 00 0). 2 On peut donc Considérer Ces deux documents Comme exprimant et disCutant de Conceptions de la qualité de la recherche en éducation.
Walsh porte un jugement sévère sur l'ensemble de Ia recherche analysée ; Celui-Ci apparaît "défiCient, peu rigoureux, vieilli et parfois malhonnête " (2 0 10, p. 13). Ce jugement se déCline en huit Critiques formulées de $\mathrm{m}$ anière polémique et catégorique. Walsh élimine un grand nombre de recherches pour des problèmes méthodologiques associés à la taille des échantillons, aux mesures utilisées, au vieillissement de la recherche $d$ ans les sCiences soCiales appliquées $m$ ais également pour des problèm es reliés à l'absence de contrôle de la production des connaissances par les membres de la Communauté scientifique. Darling-Hammond $\left(\begin{array}{lll}2 & 0 & 0\end{array}\right) 2$ estime pour sapart que s'il est légitime de soulever Ces problèmes méthodologiques, il est par ailleurs disCutable d'éliminer systématiquement tout résultat de recherche sous le prétexte que l'un ou l'autre Critère de qualité n'est pas Complètement respecté.

En fait, s'opposent iCi deux Conceptions de la qualité de la recherche en éducation, l'une qui se présente Comme restrictive et nigoureuse, éliminant une bonne partie de la production scientifique parCe que les données qu'elle utilise apparaissent "molles", subjec tives, en nombre insuffisant pour généraliser quoi que Ce soit, et l'autre, refusant d'appliquer de manière "autom atique" les Critères retenus par la première Conception, et insistant surles vertus sCientifiques de la diversité des méthodes et des niveaux d'analyse.

Ce débat intéressant est bien mené par les tenants des deux positions. Par ailleurs, ne voyant pas d'issue possible, on peut penser qu'il est donc appelé à se renouveler sans Cesse.

Quatrième proposition, formulée de manière interrogative : s'il s'avère important d'apprendre Ce métier, Comment l'apprend-t-on de manière effiCiente ? La question de politique éducative est plus directe et moins générale : les programmes de Certification alternatifs sont-ils aussi ou plus efficaces que les programmes universitaires longs?

Pour les dérégulationistes, les programmes alternatifs présentent des avantages Certains, empiriquement vérifiés. En effet, ils permettent de répondre rapidement à une situation de pénurie, dans Certaines $m$ atières, en milieu rural et en milieu ubain pauvre. Aussi, ils attirent des personnes différentes de la recrue typique d'une faculté d'éducation: Ce sont en général des adultes qui n'ont $p$ as vécu toute leur vie dans le cadre de l'institution scolaire et qui ont une expérience professionnelle riche et éventuellement stimulante pour des élèves. D'ailleurs, C.E. Finn les appelle des career transitioners et il Considère leur apport comme positif à l'éducation des jeunes, même si ce jugement relève davantage du sens 
Commun que de la recherche empirique. Selon les professionnalistes, les dérégulationistes oublient de mentionner que ce type d'enseignants a tendance à quitter l'enseignement plus rapidement que les enseignants Certifiés, prob abement parce qu'ils sont amenés à travailler dans des contextes difficiles, là où une formation plus importante s'avère néCessaire.

Par ailleurs, les dérégulationistes Citent des statistiques à l'effet que les facultés d'éduc aon recrutent leurs étudiants parmi les diplômés du secondaire les plus faibles. Le secrétaire d'État à l'Éducation du gouvernement fédéral, M. R. Paige, Cite Ces données dans son apport sur la qualité de l'enseignement $\left(\begin{array}{lll}2 & 0 & 0\end{array}\right)$ il s'en sert pour soutenir les programmes alternatifs. Darling-Hammond réplique avec des chiffres récents montrant la progression des scores des étudiants en formation des maîtres surle fameux Scholastic Aptitude Test (SAT).

De leur Côté, les professionnalistes insistent pour que, parmi l'ensemble des programmes alternatifs de Certification, des distinctions soient faites. En effet, à leurs yeux, il importe de distinguer les programmes universitaires post-undergraduate ou de maîtrise, qui attirent des étudiants détenteurs d'un baccalauréat disciplinaire, des programmes Courts offerts par le milieu scolaire, recrutant souvent des adultes désirant Changer de profession, provenant des minorités visibles et disposés à travailler dans des écoles de milieu pauvre. Dans le premier cas, on permet à des étudiants n'ayant pas choisi l'enseignement à leur entrée à l'université, de s'orienter vers l'enseignement, tout en les amenant à compléter une formation pédagogique et didactique, ainsi qu'à réussir des stages en milieu scolaire. Que Ces formations soient empiriquement démontrées efficaces réjouit et Conforte les professionnalistes dans leur position de base. Dans le second cas, il s'agit de programmes d'urgence, en vue d'assurer le recrutement d'enseignants "qualifiés»dans des matières pour lesquelles une pénurie - réelle ou Construite existe, et dans des districts scolaires et des écoles de milieux pauvres et à forte densité ethnique (afroet latino-américaine). Ces formations ne prévoient pas d'apprentissage pratique du métier avant la prise de fonction dans une école et elles ne dépassent guère la Couverture des programmes à enseigner et les informations néCessaires à l'insertion professionnelle.

Parmi ce second type de programme, le programme Teach for America, grâce au soutien du gouvernement fédéral, jouit d'une aura particulière. Pourtant, selon Laczko-Kerr et Berliner $\left(\begin{array}{lll}2 & 0 & 0\end{array}\right), 2$ les enseignants formés dans ce programme alternatif ne se sont pas révélés plus performants que les autres enseignants non-Certifiés.

Darling-Hammond élargit le débat sur les programmes alternatifs en soulignant leur contribution au maintien des inégalités sociales et scolaires. En effet, selon elle, tout se passe comme si les écoles publiques et privées, riches, situées en banlieue recrutent des enseignants Certifiés, diplômés d'un programme universitaire long, alors que les écoles publiques, des Centre-ville défavorisés et ethniquement denses, ou de milieu rural pauvre, doivent se Contenter d'enseignants non-Certifiés ou formés dans les programmes alternatifs d'urgence. En somme, deux classes d'enseignants, aux Coûts de formation différents, pour un système scolaire de plus en plus nettement dualisé. Selon Darling-Hammond, au nom de l'équité sociale, il importe de combattre cette option politique. Pour les dérégulationistes, seule l'efficacité des formations doit être Considérée.

Même si les protagonistes au débat partagent des orientations fondamentales - Ia recherche doit et peut fournir l' "évidence " nécessaire à l'élaboration des politiques de formation des enseignants et ultimement, l'efficience d'un enseignant se mesure par les gains d'apprentissage des élèves qui lui sont Confiés -, des ConCeptions différentes de l'expertise enseignante et aussi de la recherche éducative de qualité les opposent et les amènent à se livrer une lutte qui apparaît aux groupes concernés, suffisam. ment importante pour mobiliser de part et d'autre, des représentants Crédibles, voire de grandes figures (Darling-Hammond, Berliner, Floden, Ravitch, Finn, etc.). Cette mobilisation Contribue Certes à la qualité du débat, m ais aussi à sa polarisation.

\section{CONCLUSION : SORTIR DE LA POLARISATION PAR DAV ANTAGE DE CONCURRENCE, MAIS RÉGULÉE PAR L'ÉV ALUATION SCIENTIFIQUE, OU RÉINTRODUIRE LA DIMENSION SOCIALE DANS LA POLITIQUE ÉDUCATIV E}

Il est clair que l'administration am éricaine actuelle soutient la position des dérégulationistes. Dans un pareil Contexte politique, les facultés d'éducation sont placées surla défensive. Ayant accepté l'evidence. based policy et le néCessaire lien entre la formation et la réussite scolaire des élèves, elles apparaissent vulnérables et peuvent difficilement soutenir leur position monopolistique. D'ailleurs, leur discours sur le fond de la question en débat n'entre pas en 
Contradiction directe avec elui des dérégulationistes; plutôt, il Cherche à l'élargir et à le Complexifier, notamment en ce qui a trait à la Conception de l'apprentissage des élèves, de l'expertise de l'enseignant et de la qualité de la recherche. D'une Certaine façon, Cette stratégie reconnaît au moins en partie le bien-fondé de la position de l'autre. Et une fois qu'on accepte d'être sur le terrain de l'autre, il est difficile, lorsqu'en lutte, de s'en tirer indemne.

Suivant Cette ligne d'analyse, la suggestion de Finn (2 0 0 0 ) Bour sortir de la polarisation actuelle est redoutable et d'une finesse stratégique difficile à Contrer pourquiconque veut "sauver "le monopole des facultés d'éducation sur la formation des enseignants. Qu'on en juge. Finn propose aux parties de:

- Convenir d'une mesure Commune de l'efficacité des différents dispositifs de formation, à savoir la valeur ajoutée de la formation à l'apprentissage mesuré des élèves ;

- puisqu'en théorie, plusieurs dispositifs et différentes stratégies peuvent Contribuer à l'apprentissage des élèves, être ouverts d'esprit et à l'expérimentation, et non pas doctrinaires ou dogm atiques ;

- respecter les Choix des États : Ceux qui opteront pour l'agendade la professionnalisation ou pour Celui de la dérégulation devront accepter que leurs effets soient évalués à long terme, grâce à des évaluations de qualité et objectives ;

- diversifier la formation des enseignants et en évaluer les effets.

Cette proposition est séduisante, car elle se présente Comme raisonnable, pragmatique et Conforme à l'ethos politique et Culturel am éricain. Elle est aussi une voie de sortie pour les aspects scientifiques du débat puisqu'elle affirme que ce débat avancera dans la mesure où le quasi-marché de la formation se diversifiera véritablement, tout en se soumettant à une évaluation scientifique Continue et rigoureuse.

C'est aussice que soutient une Coalition d'experts dans un document soum is au Département américain de l'Éducation. Cette Coalition for EvidenceBased Policy (2 0 0 $)$ Bresse instamment le gouverne$m$ ent fédéral américain de ne soutenir financièrement que les efforts de recherche utilisant la méthode de l'expérimentation Contrôlée et aléatoire sur grande échelle (randomized trials), dans le but de Construire une base de Connaissances scientifiques. Cette base de Connaissances ne pourra devenir prescriptive, que si elle est soutenue par ce type de recherche.
Pour sa part, le doyen de la faculté d'éducation de la Northeastern University de Boston, J. Fraser $\left(\begin{array}{lll}2 & 0 & 0\end{array}\right) 2$ accepte cette idée d'un quasi-marché de la formation soum is à une évaluation de la valeur ajoutée des diplômés et tente de Convaincre ses collègues de faire de même. Selon lui, la fin du monopole aura pour Conséquence de donner plus de liberté aux universités, car elles ne seront plus tenues de se soumettre aux politiques et aux législations des États en $m$ atière $d e$ form ation $d e s$ aîtres. Elles pourront donc Construire les Curricula de formation qu'elles estimeront fondés sur l'état des Connaissances (state of the art).

II ne fait pas de doute que dans Cette voie de sortie du débat polarisé, le paradigme de l'enseignant (et $\mathrm{du}$ formateur) efficace triomphe. Ce qui apparaissait plus haut Comme une opposition forte entre l'enseignant Compétent et l'enseignant efficace s'estompe Considérablement au profit du second terme qui absorbe le premier.

Peut-il y avoir une alternative ? Il est difficile de répondre, en tout cas, on ne la voit pas émerger et se Construire dans les États-Unis actuels. À notre sens, la seule alternative possible passe par une réintroduction dans le débat d'une forte Composante d'une idéologie socialement Connotée et d'une Critique à la fois des institutions actuelles de formation et des thèses de dérégulationistes pour leur Contribution aux inégalités sociales et à la hiérarchisation des écoles. Le risque Cependant est alors de sembler vouloir opposer la science ou la recherche et l'idéologie. Peut-être pas, car rien n'empêche les professionnalistes de travailler à la construction de bases de données de nature à soutenir leur thèse. Après tout, Bourdieu et les autres théoriciens de la reproduction ont élaboré leur théorie en s'appuyant sur des analyses empiriques rigoureuses.

Le débat américain sur la formation et la Certification des enseignants nous force à réfléchir surles rapports entre la science et l'idéologie. Certes, les $p$ artis prennent très au sérieux Ce débat quidure depuis maintenant deux décennies. Ils y ont investi une importante capacité de mobilisation et de réseautage, ont entrepris de nombreuses études empiriques et Complété des méta-analyses des recherches existantes. Ils ont même Contribué à raffiner Certaines méthodologies appropriées à l'étude de l'effetenseignant, et ils ont Construit des argumentaires serrés nommant les points d'accord et de désacCords fondamentaux entre les partis. Il y a là un véritable débat (8). 
Paradoxalement, le déb a montre que plus on Cherche à évacuer l'idéologie du déb a considérant toute référence idéologique illégitime dans une volonté de ne soumettre l'élaboration des politiques éduc aves qu'àdes résultats scientifiques "incontestables", plus on lie en quelque sorte la science à une idéologie particulière qui refuse de se nommer Comme telle. Car les dérégulationistes, dévoués à ıa sCience de la mesure empirique nigoureuse, épousent néanmoins une Conception de l'apprentissage qui n'est pas neutre et ils ont une vision de l'expertise enseignante et de la formation requise pour la développer qui participe du modèle séculaire de l'artisan instruit (Paquay, 1994). En Ces matières, il ne peut $p$ as ne pas y avoir de référence à des valeurs, à des Conceptions du désirable.

La Conception des dérégulationistes à propos de la relation entre la science et la politique est étrangement silencieuse sur les inévitables et nécessaires médiations idéologiques entre, d'une part, Ia science et les jugements de fait, et d'autre part, Ia politique et les jugements de valeur. En fait, Cette Conception doit être qualifiée d'étroite et de réductrice, puisqu'en somme, la thèse fondamentale consiste à soutenir que l'État ne doit élaborer de politiques Contraignantes pour les acteurs et imposer des pratiques que si la science incontestablement soutient dans les faits ses prétentions. Sinon, il doit s'abstenir et Iaisser faire. Cela est évidemment un peu Court et du même souffle, d'une prétention hégémonique : seule la science peut trancher des questions litigieuses, transcender les intérêts particuliers et incarner le bien Commun. C'est parce que la science est imparfaite ou insuffisamment développée que la politique est soum ise àdes effets de mode, des intérêts "Corporatistes" ou des tendances idéologiques. Pour Contrer Ces modes et Ces dérives, il faut donc développer la science, Celle qui produit des jugements de fait "inContestables". Ainsi l'evidence-b aed policy se développera et rassemblera tous les acteurs autour d'un bien Commun sCientifiquement défini.

Loin de nous l'idée que la recherche évaluative soit inutile ou que l'eviden $c e-b$ aed policy soit à bannir. C'est plutôt le piège qu'elles Comportent qui doit être soum is à la Critique. Ce piège, C'est de réduire l'apprentissage à ce qui est mesurable, l'expertise enseignante à son efficacité, conçue Comme valeur ajoutée, et la valeur de l'éducation à son instrumentalité. Pour éviter Ce piège, il faut rappeler la position wébérienne de l'irréductibilité des types de jugements.

Claude Lessard claude.lessard@montreal.ca Université de Montréal

Faculté des sciences de l'éducation

\section{NOTES}

(1) Même si la plupart des enseignants des éColes privées améri caines sont de fait Certifiés, les écoles privées ne sont pas obligées d'em baucher des enseignants Certifiés. Seules les écoles publiques doivent se soum ettre à Cette exigence.

(2) Linda Darling-Ham mond est professeur d'éducation à ıa Faculté d'éducation de I'Université Stanford en Californie. Elle est Ia directrice générale de la National Commission on Teaching and Am erica's Future (NCTAF).

(3) Diane Ravitch est présentement research professor à l'université New York. Au Cours des années 1980 , elle fut asso Ciée au travail du groupe d'experts à l'origine du Célèbre apport $A$ Nation at Risk. Au Cours des années 1990 , elle a été assistante au secrétaire d'État à l'Éducation et fut responsable de la direction de la recherche et du développement en éducation du Département am éricain (fédéral) de l'Éducation. Elle est m embre de la Ford $h$ am Foundation qui défend l'agenda de la dérégulation de l'éducation, en général, et dans le domaine de la formation des enseignants, en particulier.

(4) Ardent défenseur de la dérégulation en éducation, C. E. Finn détient un doctorat en politique éducative de Harvard. Il a été professeur d'éducation et de politique publique à l'université Vanderbilt pendant vingt ans. Tout Comme D. Ravitch, il fut assistant secrétaire de l'Éducation et fut responsable de la direction de la recherche et du développement en éducation du Département américain (fédéral) de l'Education et a signé le rapport A Nation at Risk (1983). Au Cours des années, il a travaillé pour plusieurs fondations et il préside présentement, entre autres, le Thom as B. Fordham Foundation et le Thom as B. Fordham Institute. Il a également participé à la fondation du "Projet Edison", projet de prise en Charge par l'entreprise privée de districts sColaires et des écoles publiques qu'ils Comprennent et de mise sur pied d'une administration obéissant aux principes du nouveau m an agem ent public.

(5) Chaque État dem eure libre de lég iférer Com me il l'entend en Cette m atière et d'ajouter d'autres éléments de Certification. Il doit Cependant s'assurer que les enseignants qu'il Certifie possède une Compétence dis Ciplinaire

(6) En annonçant la création de la Commission, le secrétaire Bell déclara qu'il était préoccupé du "widespread public perception that something is seriously remiss in our educational system ". On peut d'ailleurs penser que la forte présence d'universitaires dis Ciplinaires (y Compris les présidents d'université) dans la Comm is. sion explique en partie la volonté du rapport de "tirer le high school américain vers le haut", C'est-à-dire d'en accroître les exigences et la qualité en grande partie en fonction des besoins de l'enseignement supérieur.

(7) Pour présenter un portrait Complet des idéologies qui s'affrontent, un troisième Courant devrait être présenté : C'est Celui qui met l'accent surla liberté de choix de l'école par les parents, Ia concurrence entre établissements, et l'imputabilité et la reddition de Comptes, bref, les avancées en éducation du New Public Manage ment. Ni A Nation at Risk, ni A Nation Prepared ne s'inspirent direCtement de Ce type de disCours, encore que Celui-Ci ne soit $\mathrm{p}$ as in Compatible avec le discours de A Nation at Risk. Cependant, il heurte de front l'agenda de la professionnalisation, substituant au Contrôle par laprofession, la régulation du m arché éducatif.

(8) Davantage qu'un Combat, au sens que BourdonCle (2 0 10) donnait à ce terme en référant au contexte français. 


\section{BIBLIOGRAPHIE}

BouRdoncle R. (2001) «La carte de la pensée et le Champ du débat". Revue française de pédagogie, n० 135 , p. $61-64$.

Carnegie Forum on Education and The Economy (1986). $A$ Nation Prepared: Teachers for the 21st Century, The Report of the Task ForCe on Teaching as A Profession. New York.

Coalition for Evidence-Based Policy (2002). Bringing EvidenCe-Driven Progress to Education: A ReCommended Strategy for the U.S. Department of Education. Docum ent multigraphié, disponible sur Internet au format PDF à l'adresse: http://Coexgov.seCuresites.net/admin/ Form Manager/filesuploading/Coalition Fin Rpt.pdf (Consulté le 22 février 2006 ).

Cochran-Smith M. \& Fries M. K. (2001). "Sticks, Stones, and ldeology : the Discours of Reform in Teacher Education". Educational Researcher, vol. 30, n8 8, p. 3-15.

Coleman J. S. et al. (1966). Equality of Edu cational Opportunity. Washington [DC] : Government Printing Office.

Darling-Hammond L. (2 0 0). 0“ Teacher Quality and Student Achievement : A Review of State Policy Evidence". Education Policy Analysis Archives, vol. 8, n० 1. Disponible sur Internet à l'adresse : http://epaa.asu.edu/ epaa/v8n1/ (Consulté le 22 février 2006).

Darling- Hammond L. (2002). "Research and Rhetoric on teaCher Certification: A response to "Teacher Certification ReConsidered" ". Education Policy Analysis ArChives, vol. $10, n^{\circ} 36$. Disponible sur Internet àl'adresse : http :// epaa.asu.edu/epaa/v10n36/ (Consulté le 22 février 2006).

Darling-Hammond L. ; Chung R. \& Frelow F. (2002). "Variation in Teacher Preparation, How Well Do different Pathways Prepare Teachers to Teach ?". Journal of Teacher Education, vol. 53, n 4, p. 286-302.

De Broucker P. \& Sweetman A. (2002). Tow ards EvidenceBased Policy for Canadian Education, Vers des politiques canadiennes d'éducation fondées sur la reCherChe. Montréal: Kingston: John Deutsch Institute for The Study of Economic Policy; Queen's University et Statistique Canada; McGill-Queen's University Press.

FEISTRITZER C. E. (2004). Alternatives Teacher Certification. A State by State Analysis. Washington [DC] : National Center for Education Inform ation.

FINN C. E. (2003). "Teacher Reform Gone Astray". In P. E. Peterson (éd.), Our Schools and Our Future... Are we Still at Risk? Stanford: Hoover Institution Press Publication, $n^{\circ} 516, p .211-238$.

FLeXNeR A. (1910). Medical Education in the United States and Canada. Carnegie Forum on Education and The Econom y Bulletin $n^{\circ} 4$.
Fraser J. (2002). "A Tenous Hold". Education Next.

GoldhabeR D. (2002). "The Mystery of Good Teaching". Education Next, vol. 2, n०1, p. 50-55.

HESS F. M. (2001). Tear Down this Wall, the Case for a Radical Overhaul of Teacher Certification, Progressive Policy Institute, $21^{\text {st }}$ Century sChool projeCt.

HITE S. J. (2001). Reviewing quantitative Research to inform Educational Policy Processes. Paris : Unesco Institute for Educational Pianning .

LACZKo-KerR I \& BerLineR D. C. (2002). "The effectiveness of "teach for America" and other unbder-Certified Teachers on student academic Achievement : a case of harmful public policy". Education Policy Analysis Archives, vol. 10, n० 37. Disponible sur Internet à l'adresse : http://epaa.asu.edu/epaa/v10n37/ (Consulté le 22 février 2006).

PAQUAY L. (1994). "Vers un référentiel de Compétences professionnelles de l'enseignant? ", ReCherche et forma tion pour les professions del'éducation, n॰16, p. 7-38.

Ravitch D. (2003). "A Historic Document". In P. E. Peterson (éd.), Our Schools and Our Future... Are we Still at Risk? Stanford: Hoover Institution Press Publication, n०516, p. $25-38$.

United States of AMERICA : National COMMitTeE on EXCEllence IN EducATION (EXCELLENCE COMMISSION) (1983). A Nation at Risk. Washington [DC] : Departm ent of Education.

United States of America : National Commission on Teaching AND AMERICA'S FUtURE (1996). What Matters Most : TeaChing for Am erica's Future. New York : NCTAF.

United States Of America : Education Commission of the STATES $(2000)$. Quality Teaching: Two Paths to Quality Teaching: Implications For Policymakers. Document disponible sur Internet à l'adresse : http://www.eCs. org/Clearinghouse/12/22/1222.htm (Consulté le 22 février 2006 ).

United States of America : Department of Education (2 0002 ). Meeting the Highly qualified Teachers Challenge. The Secretary's Annual Report on Teacher Quality. Washington [DC]: Office of Postse Condary Education.

United States of America : Department of Education (2 0003 ). ldentifying and Implementing Educational Practices Supported By Rigorous Evidence: A User Friendly Guide. Washington [DC] : Institute of Education Sciences, National Center for Education Evaluation and Regional Assistan Ce.

WALSH K. (2001). Teacher Certification Reconsidered: Stum bling for Quality. The Abell Found ation.

WALSH K. (2002). "Positive spin, the evidence for traditional teacher Certification reexamined ", Education Week. 